\title{
Superconductivity due to cooperation of electron-electron and electron-phonon interactions at quarter filling
}

\author{
R. T. Clay (1)* and D. Roy (1) \\ Department of Physics \& Astronomy and $\mathrm{HPC}^{2}$ Center for Computational Sciences, Mississippi State University, \\ Mississippi State, Mississippi 39762, USA
}

(Received 13 November 2019; revised manuscript received 27 February 2020; accepted 11 March 2020; published 3 April 2020)

\begin{abstract}
Existing theories of superconductivity emphasize either electron-electron or electron-phonon interaction, each of which tends to cancel the effect of the other. We present direct evidence from unbiased numerical calculations of cooperative, as opposed to competing, effects of electron-electron and electron-phonon interactions within the frustrated Hubbard Hamiltonian with bond-coupled phonons, uniquely at the band filling of one-quarter. The two interactions cooperatively reinforce $d$-wave superconducting pair-pair correlations at this filling while competing with one another at all other densities. Our work gives insight into how intertwined charge order and superconductivity appear in real materials.
\end{abstract}

DOI: 10.1103/PhysRevResearch.2.023006

\section{INTRODUCTION}

There is strong evidence that any successful theory of superconductivity (SC) in the high- $T_{c}$ cuprates and other unconventional superconductors must incorporate the effects of electron-electron $(e-e)$ interactions. It is now often assumed that superconducting pairing in these materials can be mediated solely by $e-e$ interactions, leading to numerous studies of model Hamiltonians with $e-e$ interactions such as the Hubbard model. While the properties of the two-dimensional (2D) Hubbard model remain under active debate, calculations with the best unbiased methods available have found no long-range superconducting pairing in the weakly doped Hubbard model on a square lattice [1,2] or the half-filled Hubbard model on the triangular [3] and anisotropic triangular [4] lattices. These calculations also find that pairing correlations become weaker with increasing $e-e$ interaction strength [1-4].

One possible reason calculations have failed to find SC is that repulsive $e$ - $e$ terms alone are not sufficient for $\mathrm{SC}$ and other interactions are required. The only other interaction common to all unconventional superconductors is electronphonon $(e-p)$ coupling. In the cuprates there is strong experimental evidence for the importance of phonons in the electronic properties and SC. This includes giant softening of the $\mathrm{Cu}-\mathrm{O}$ bond stretching frequency in the underdoped cuprates [5], the oxygen isotope effect [6,7], and kinks observed in photoemission experiments [8]. Phonons also play a role in the ubiquitous charge ordering found in all cuprates [5,9].

\footnotetext{
*r.t.clay@msstate.edu

Published by the American Physical Society under the terms of the Creative Commons Attribution 4.0 International license. Further distribution of this work must maintain attribution to the author(s) and the published article's title, journal citation, and DOI.
}

Charge ordering in cuprates and other unconventional superconductors has led to proposals that correlated-electron SC evolves from or is intertwined with spatial broken-symmetry states, for example, density waves of Cooper pairs [10-15]. It is however not clear what such pair-density-wave states look like in detail or emerge from.

A key theoretical idea behind the belief that $e$ - $e$ interactions mediate SC in the nearly half-filled band is the resonatingvalence-bond (RVB) theory of SC, in which SC is proposed to emerge from a proximate half-filled spin-gapped valencebond solid (VBS) state [16]. While this idea is both promising and exciting, in addition to the lack of evidence for SC in the nearly-half-filled Hubbard model [1-4], theoretical evidence for the VBS within 2D models is sparse or absent. At half filling, a VBS would be most likely to be found on frustrated lattices in the strong interaction limit of the Hubbard model, the Heisenberg model. A VBS ground state is found in the 2D Heisenberg model in the presence of strong four-spin interactions [17]. However, in the $J_{1}-J_{2}$ Heisenberg model, while some recent numerical results suggest the possibility of a VBS phase between the Néel and stripe antiferromagnetic (AFM) phases [18], other calculations find no VBS order $[19,20]$. In the frustrated half-filled Hubbard model [3,21] or Hubbard-Heisenberg model [22] numerical studies to date have not found evidence for a VBS phase.

Singlet formation is favored over AFM order in one dimension, and a 2D system can be driven to a singlet phase by making the system effectively one dimensional through segregated stripes of charges. In this case the density must be less than one charge per site. Clay and co-workers have proposed a valence-bond theory of SC that combines features of the Cooper pair-density-wave concept and RVB theories, with the critical difference that the theory applies uniquely to the quarter-filled band (i.e., carrier density $\rho=0.5$ ) [23]. At $\rho=0.5$ in the presence of lattice frustration, a paired-electron crystal (PEC) emerges that is a density wave of spin singlets, 
with nearest-neighbor (NN) singlet pairs separated by pairs of vacant sites. The PEC is the quarter-filled equivalent of the VBS, characterized by coexisting spin gap and charge or bond order, and is favored over AFM order in the presence of lattice frustration $[24,25]$.

Initial theoretical evidence for a correlated $\rho=0.5 \mathrm{su}-$ perconducting state within the electron-only Hubbard Hamiltonian has been presented [26-28]. Strong experimental evidence exists as well in the organic charge-transfer solid (CTS) superconductors, all of which are $\rho=0.5$ [23]. In the CTS the PEC is found proximate to $\mathrm{SC}$ with pressure taking the place of doping [23]. Besides the CTS, many $\rho=0.5$ superconductors are known [23]. The theory has been further generalized to the cuprates, where it has been suggested that, following a valence transition, the oxygen $p$ orbitals become the active sites in the $\mathrm{Cu}-\mathrm{O}$ planes, forming a frustrated $\rho=$ 0.5 lattice [29]. It is important to note that $e$ - $p$ interactions are required to realize the $\mathrm{PEC}$, because bond distortions must occur simultaneously with NN singlet formation at $\rho=0.5$ $[24,25]$. If SC indeed emerges from the destabilization of the PEC, it has to be driven by both $e-e$ and $e-p$ interactions. This is what we will demonstrate in the present paper. In Sec. II we discuss previous work on the effect of combined $e-p$ and $e-e$ interactions and introduce the model we study. Section III introduces the method and numerical results and Sec. IV summarizes our findings.

\section{MODEL}

In most theories $e-p$ and $e-e$ interactions have been considered to be mutually exclusive in mediating SC. This belief is partly due to the failures of the phonon-based pairing mechanism of the BCS model in unconventional superconductors [30], but is also reinforced by the study of Hamiltonians that combine $e-e$ and $e-p$ interactions. In many of the models studied to date, phonons are assumed to only couple to the local charge density. In general, the effects of these types of phonons will compete with the Hubbard $U$, which prevents double occupancies. The simplest system is the HubbardHolstein model (HHM), where at each site a dispersionless phonon is coupled to the charge density:

$$
\begin{aligned}
H= & -\sum_{\langle i, j\rangle, \sigma} t_{i j}\left(c_{i, \sigma}^{\dagger} c_{j, \sigma}+\text { H.c. }\right)+U \sum_{i} n_{i, \uparrow} n_{i, \downarrow} \\
& +g \sum_{i, \sigma} x_{i} n_{i, \sigma}+\sum_{i}\left(\frac{p_{i}^{2}}{2 M}+\frac{M \omega^{2}}{2} x_{i}^{2}\right) .
\end{aligned}
$$

In Eq. (1) $c_{i, \sigma}^{\dagger}$ creates an electron of spin $\sigma$ on site $i, n_{i, \sigma}=$ $c_{i, \sigma}^{\dagger} c_{i, \sigma}, t_{i j}$ is the electron hopping integral, and $U$ is the on-site Hubbard interaction. In addition, $x_{i}$ and $p_{i}$ are coordinate and momentum operators for the phonon oscillator at site $i$ with mass $M$ and frequency $\omega$. The $e-p$ coupling constant is $g$. We give energies below in units of the bare hopping $t$. The physics of the HHM is governed by the competition between on-site pairing driven by the $e-p$ interaction and opposed by $U$. This is most clearly seen in the antiadiabatic limit $(M \rightarrow 0, \omega \rightarrow \infty)$ where the effective Hubbard interaction between electrons is $U_{\text {eff }}=U-2 g^{2} / \omega$.
Phonons also couple to the kinetic energy of electrons. We consider here a model with dispersionless bond-coupled (SuSchrieffer-Heeger-type [31]) phonons, the Peierls-Hubbard model

$$
\begin{aligned}
H= & -\sum_{\langle i, j\rangle, \sigma} t_{i j}\left[1+\alpha x_{(i j)}\right]\left(c_{i, \sigma}^{\dagger} c_{j, \sigma}+\text { H.c. }\right) \\
& +U \sum_{i} n_{i, \uparrow} n_{i, \downarrow}+\sum_{\langle i, j\rangle}\left(\frac{p_{(i j)}^{2}}{2 M}+\frac{M \omega^{2}}{2} x_{(i j)}^{2}\right) .
\end{aligned}
$$

In Eq. (2) $x_{(i j)}$ is the phonon coordinate associated with the deformation of the bond connecting sites $i$ and $j$ and all the other terms have identical meaning to Eq. (1). Compared to the large amount of work on the HHM, there are few numerical studies of bond-coupled phonons beyond the classical limit. Most work at finite $\omega$ has been in one dimension and mostly at $\rho=1$ (see Ref. [32] for a recent review) [33-36]. A 2D multiorbital lattice was studied recently, although only for $U=0$ [37].

Even for $U=0$ the antiadiabatic limit of Eq. (2) results in a much more complex effective interaction between electrons than in the HHM [36,38-41]. For Eq. (2) on a 2D square lattice the effective Hamiltonian $H_{\mathrm{W}}$ has the form [39-41]

$$
H_{\mathrm{W}}=-W \sum_{i} K_{i}^{2},
$$

where $W=\frac{2 \alpha^{2}}{M \omega^{2}}$ and $K_{i}$ is the kinetic energy of bonds connected to site $i$,

$$
K_{i}=\sum_{\sigma, \vec{\delta}}\left(c_{\vec{i}+\vec{\delta}, \sigma}^{\dagger} c_{\vec{i}, \sigma}+\text { H.c. }\right),
$$

with $\vec{\delta}=\{+\hat{x},+\hat{y},-\hat{x},-\hat{y}\}$. There are four types of terms in Eq. (3): a chemical potential term, nearest-neighbor repulsion for parallel spins, on-site pair terms (which will be suppressed by $U)$, and pair hopping of nearest-neighbor singlet pairs [36,38-41]. Recent work has shown that the pair hopping interaction can lead to strongly bound bipolarons with light effective masses that are stable against strong $e$ - $e$ interactions [36]. This suggests the possibility of $e-p$ mediated SC in the presence of strong $e-e$ interactions at finite carrier densities.

The effect of adding $H_{\mathrm{W}}$ to the 2D Hubbard Hamiltonian was studied using ground-state and finite-temperature quantum Monte Carlo near $\rho=1$ [39-41]. Long-range $d_{x^{2}-y^{2}}$ superconducting order was found for sufficiently strong $H_{\mathrm{W}}$ in the ground state, with a transition from AFM order to $d_{x^{2}-y^{2}}$ $\mathrm{SC}$ as the strength of $H_{\mathrm{W}}$ is increased [39-41]. However, as in the HHM, here again the SC state was suppressed by $U$. While this shows that $d$-wave SC can in principle result from $e$ - $p$ interactions, it suggests that $d$-wave SC is only possible provided the $e-p$ coupling is strong enough to overcome $e-e$ interactions, again reinforcing the belief that $e-e$ and $e-p$ interactions cannot simultaneously drive SC. Importantly, $H_{\mathrm{W}}$ and Eq. (2) have not been investigated in two dimensions at densities significantly different from $\rho=1$.

Below we study Eq. (2) directly using quantum Monte Carlo over a wide density range. While being consistent with previous results for the effective model at $\rho \approx 1$, our results show that the behavior at $\rho=0.5$ is qualitatively different 
from other densities and that at $\rho=0.5, e-e$ and $e-p$ interactions can cooperatively enhance pairing.

\section{NUMERICAL RESULTS}

We define singlet pair creation operators $\Delta_{i}^{\dagger}$,

$$
\Delta_{i}^{\dagger}=\sum_{\nu} \frac{g(\nu)}{\sqrt{2}}\left(c_{i, \uparrow}^{\dagger} c_{i+\vec{r}_{v}, \downarrow}^{\dagger}-c_{i, \downarrow}^{\dagger} c_{i+\vec{r}_{v}, \uparrow}^{\dagger}\right),
$$

where $g(v)$ is a relative sign that determines the pairing symmetry. We consider four types of pairing: (i) NN pairs with $\vec{r}_{v}=\{+\hat{x},+\hat{y},-\hat{x},-\hat{y}\}$ and $g(v)=\{+1,+1,+1,+1\}$ or $\{+1,-1,+1,-1\}$ for $s$ or $d_{x^{2}-y^{2}}$ pairing, respectively, and (ii) next-nearest-neighbor pairs with $\vec{r}_{v}=\{\hat{x}+\hat{y},-\hat{x}+\hat{y},-\hat{x}-$ $\hat{y}, \hat{x}-\hat{y}\}$ and $g(v)=\{+1,+1,+1,+1\}$ or $\{+1,-1,+1,-1\}$ for $s_{x y}$ or $d_{x y}$ pairing, respectively.

The pair-pair correlation function is $P(r)=\left\langle\Delta_{i}^{\dagger} \Delta_{i+\vec{r}}\right\rangle$. A theory of correlated electron SC should satisfy two requirements: (i) At zero temperature $P(r)$ must have longrange order and (ii) $P(r)$ in the presence of $e$-e interactions should be enhanced over its value for noninteracting fermions. Finite- and zero-temperature calculations on frustrated Hubbard models (with no $e-p$ coupling) of up to 128 sites have shown that $U$ enhances $d$-wave pairing preferentially at $\rho=$ 0.5 while suppressing pair-pair correlations relative to their $U=0$ value at all other $\rho$ [26-28]. While enhanced by $U$ the magnitude of $P(r)$ however decreases with distance, consistent with either a zero or possibly small long-range superconducting order parameter [26-28].

To solve Eq. (2) we use finite-temperature determinant quantum Monte Carlo (DQMC), which provides unbiased results [42]. The lowest temperatures that can be reached in DQMC are however limited by the fermion sign problem. In the density region of most interest here, $\rho \approx 0.5$, the sign problem is considerably less severe than for the more intensively studied $\rho \approx 0.8$. Nevertheless, we are not able to reach low enough temperatures to determine whether the ground state has long-range superconducting order. It is important to recall that the effective model $H_{\mathrm{W}}$ does have long-range superconducting order in the ground state [39-41]. Besides the sign problem, Monte Carlo autocorrelation times often increase exponentially with $e-p$ coupling near phase transitions [43]. To help mitigate this we implemented the block phonon updates of Ref. [44]. We use an imaginary time discretization of $\Delta \tau=0.05$, which is small enough that this source of systematic error can be neglected. We report results in terms of the dimensionless $e-p$ coupling strength $\lambda=\alpha^{2} t_{x} / M \omega^{2}$ and set $M=1$. Further information on the method, including comparisons with other methods in one dimension, the autocorrelation time of the method, and the dependence on $\Delta \tau$, is given in the Supplemental Material [45].

We performed calculations on $4 \times 4,6 \times 6$, and $10 \times 10$ anisotropic triangular lattices [26]. This lattice has a single frustrating bond $t^{\prime}$ across each plaquette; in the limit $t^{\prime}=t$ $\left(t^{\prime}=0\right)$ it is the triangular (square) lattice. We assume $e-p$ coupling only to the $t_{x}$ and $t_{y}$ bonds. Several families of organic CTS superconductors ( $\alpha, \beta, \beta^{\prime}$, and $\beta^{\prime \prime}$ structures) have very similar tight-binding lattice structures [23]. Because

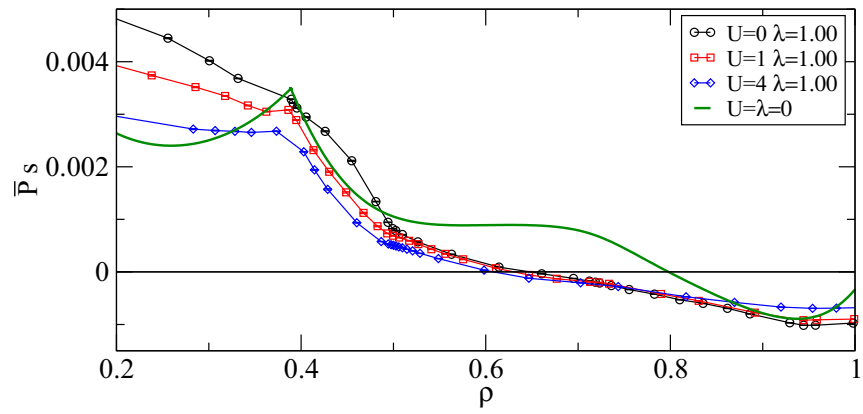

FIG. 1. Average $s$ long-range pair-pair correlations $\bar{P}$ versus density $\rho$ for the $6 \times 6$ anisotropic triangular lattice with $\omega=0.5$ at inverse temperature $\beta=8$.

the PEC requires lattice frustration [24,25], we work in the strongly frustrated limit with $t^{\prime}=0.8$. The lattice dimensions are chosen under the constraint that the $\rho=0.5$ singleparticle state is nondegenerate [26]. We also take $t_{y}=0.9$ slightly different from $t_{x}=1.0 \mathrm{in}$ order to increase the number of nondegenerate densities. This lessens the severity of the fermion sign problem and makes calculations feasible over a range of $\rho$. The precise choice of $t_{x}, t_{y}$, and $t^{\prime}$ is however not critical to the results we report.

$P(r=0)$ can be decomposed into combinations of charge and spin correlations [46]. Antiferromagnetic order leads to a trivial increase of the short-range component of $P(r)$, even as the long-range component is strongly suppressed $[4,46]$. To mitigate such finite-size effects we exclude small- $r$ correlations and measure the average long-range value of $P(r)$ [47],

$$
\bar{P}=N_{p}^{-1} \sum_{r>2} P(r) .
$$

In Eq. (6) only correlations over distances greater than two lattice spacings are kept; $N_{p}$ is the number of such terms.

For the $4 \times 4$ and $6 \times 6$ lattices we calculated $\bar{P}$ over the density range $0.2 \lesssim \rho \leqslant 1.0$. In Fig. 1 we plot $\bar{P}$ for $s$ pairing versus density for the $6 \times 6$ lattice (similar data for the $4 \times 4$ lattice and for $s_{x y}$ pairing are in the Supplemental Material [45]). We find that at all densities $U$ suppresses $s$ pairing; as $U$ increases $\bar{P}$ becomes closer to zero across the entire density range. There is an increase in $\bar{P}$ for $s$ pairing over its value for noninteracting electrons for strong $\lambda$ in the low-density region $\rho \lesssim 0.4$. An $s$ or $s_{x y}$ SC state may exist in the model in the very-low-density region provided $U$ is not too large; we will not consider this parameter region further here. The $s_{x y}$ pairing shows some enhancement by $\lambda$ for $\rho>0.4$, but is also suppressed by $U$ [45].

In the thermodynamic limit on the anisotropic triangular lattice we expect a pairing symmetry that mixes $d_{x y}$ and $d_{x^{2}-y^{2}}$; on finite lattices either $d_{x^{2}-y^{2}}$ or $d_{x y}$ is favored [26,48]. In Fig. 2 we plot $\bar{P}$ for the $d_{x y}$ symmetry versus density for the $6 \times 6$ lattice. Figure 2(a) shows the effect of increasing $U$ at fixed $e-p$ coupling strength. Compared to the noninteracting system (solid line), pairing correlations are enhanced by $U$ selectively at $\rho=0.5$ [26-28]. At all other densities pairing is suppressed by $U$. Figure 2(b) shows the effect of increasing $e-p$ coupling at fixed $U$. Here phonons enhance the pairing over a wide 


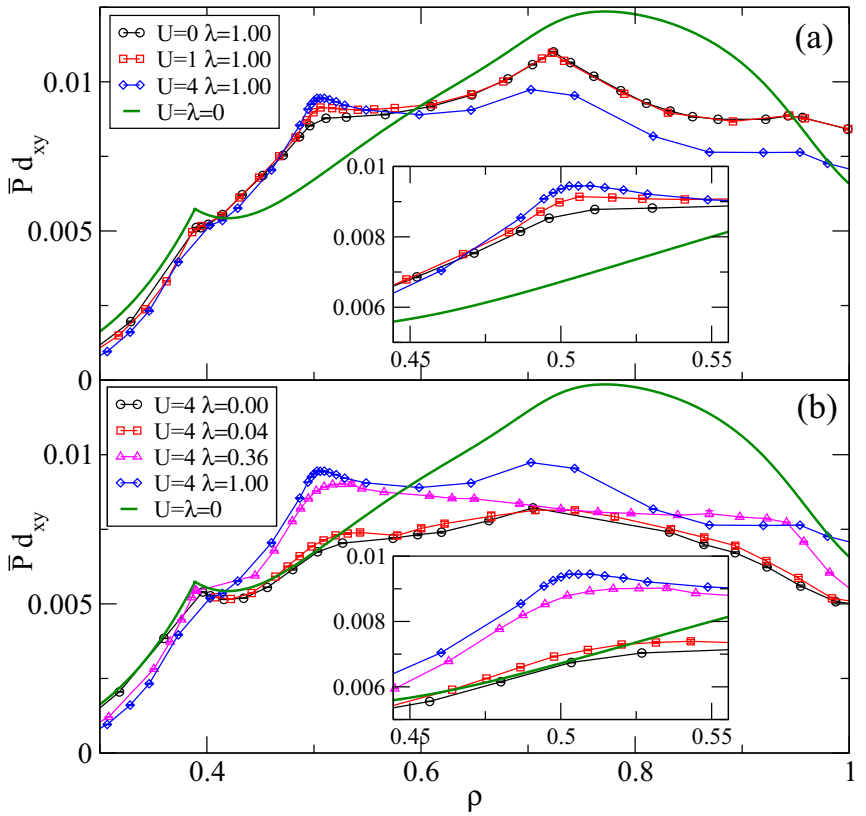

FIG. 2. Same parameters as in Fig. 1 but for $d_{x y}$ pairing: (a) effect of increasing $U$ at fixed $\lambda$ and (b) effect of increasing $\lambda$ at fixed $U$. Both interactions enhance $d_{x y}$ pairing at $\rho=0.5$. The insets magnify the density region around $\rho=0.5$.

density range for $\rho \gtrsim 0.4$, including at both $\rho=0.5$ and $\rho=1$. However, only at $\rho=0.5$ do $e-e$ and $e$ - $p$ interactions both enhance $\bar{P}$; at other densities the interactions compete.

For the $10 \times 10$ lattice we find that the fermion sign is reasonable for both $\rho \approx 0.5$ and $\rho=1$. Figure 3 summarizes our results for $\rho=1$ on all of the lattices. At $\rho=1, \bar{P}$ for either $d_{x^{2}-y^{2}}$ or $d_{x y}$ pairing increases with $\lambda$ for $\lambda \lesssim 1$. This is consistent with the $d$-wave superconducting state found in Refs. [39-41]. However, $U$ competes with the $e-p$ interaction at $\rho=1$ with $\bar{P}$ decreasing with increasing $U$. We expect on

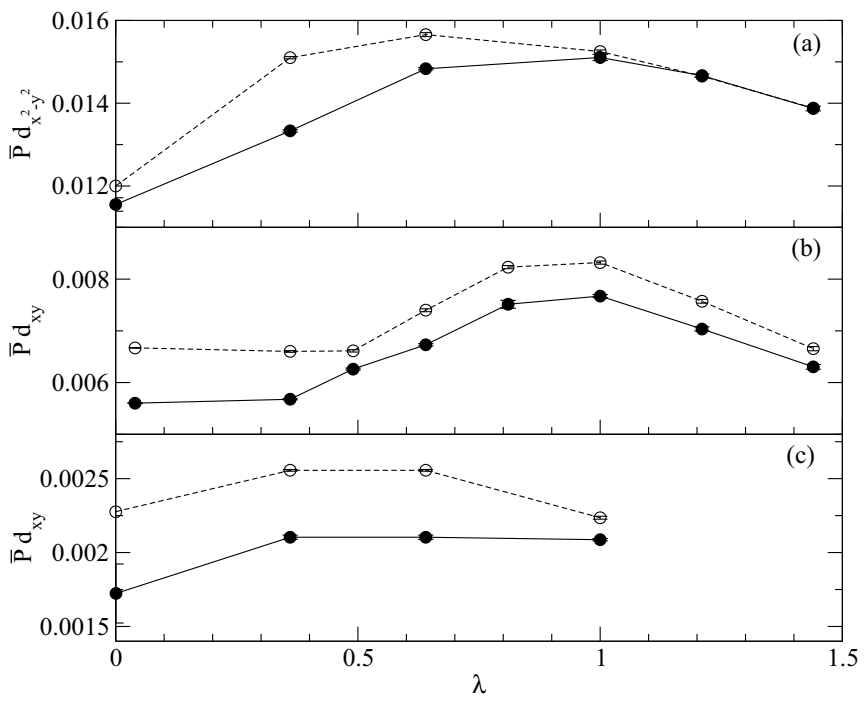

FIG. 3. Plot of $\bar{P}$ versus $e$ - $p$ coupling strength $\lambda$ for $\rho=1, \beta=$ 8 , and $\omega=0.5$ on the (a) $4 \times 4$, (b) $6 \times 6$, and (c) $10 \times 10$ lattices. Open (closed) symbols are for $U=0(U=3)$.

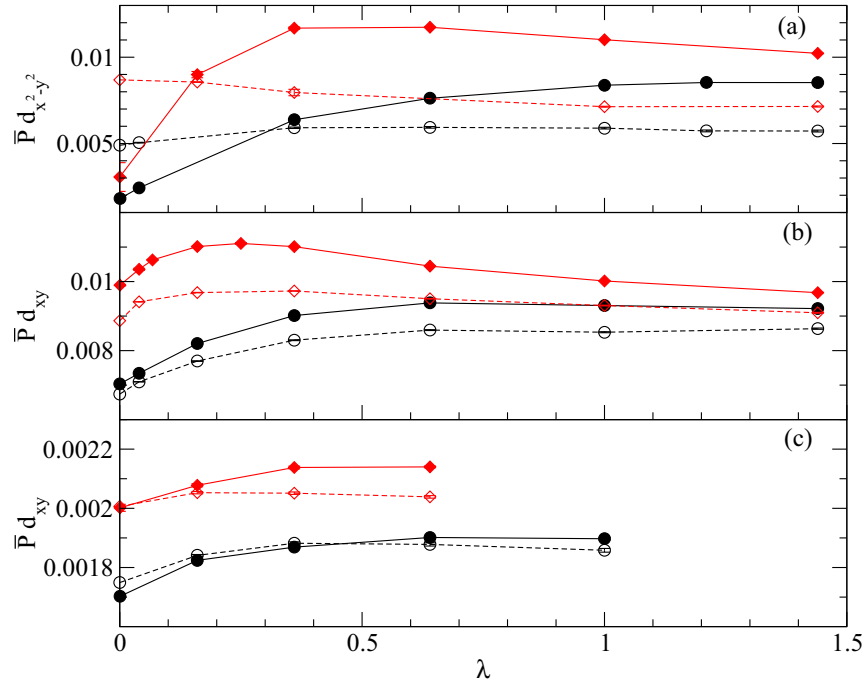

FIG. 4. Plot of $\bar{P}$ versus $e$ - $p$ coupling strength $\lambda$ for $\rho \sim 0.5$ and $\omega=0.5$ on the (a) $4 \times 4(\rho=0.375)$, (b) $6 \times 6(\rho=0.500)$, and (c) $10 \times 10(\rho=0.460)$ lattices. Circles (diamonds) are for the inverse temperature $\beta=8(\beta=16)$. Open (closed) symbols are for $U=0(U=3)$.

less frustrated lattices at $\rho=1$ a competition between $d$-wave $\mathrm{SC}$ mediated by the bond phonons and AFM order mediated by $U$. On the $4 \times 4$ lattice at large $\lambda, \bar{P}$ for $s_{x y}$ pairing becomes comparable to $\bar{P}$ for $d_{x^{2}-y^{2}}$ pairing [45]. This may be the reason for the weak decrease of the $d_{x^{2}-y^{2}} \bar{P}$ with $U$ at large $\lambda$ in Fig. 3(a).

The behavior of $\bar{P}$ at $\rho \approx 0.5$ (Fig. 4) is very different from $\rho=1$. At $\rho \approx 0.5$ zero-temperature calculations on the Hubbard model $(\lambda=0)$ find that $\bar{P}$ increases with $U$, with the density deviation from $\rho=0.5$ decreasing with increasing lattice size [26-28]. Here, for all the lattices, we find that $\bar{P}$ increases with increasing $U$ and $\lambda$ at the same densities where $T=0$ calculations find enhancement by $U$ alone. Cooperative interactions, however, should not merely both increase the value of an order parameter, but the effect of the first interaction should be strengthened in the presence of the second and vice versa. This is indeed what we see at $\rho=0.5$. First, we see that $\lambda$ enhances the effect of $U$ : In Fig. 4 the increase in $\bar{P}$ between $U=0$ and $U=3$ for all lattices is larger for $\lambda>0$ than at $\lambda=0$. In fact, at the temperatures we can access here, on some lattices $\bar{P}$ decreases with $U$ at $\lambda=0$. Second, nonzero $U$ enhances the increase in $\bar{P}$ with $\lambda$. As a function of $\lambda, \bar{P}$ reaches a broad maximum at $\lambda=\lambda_{\max }$. Comparing the values of $\bar{P}$ at $\lambda=0$ and $\lambda_{\max }$, there is a larger increase for $U>0$ compared to $U=0$. These data show that at $\rho \approx 0.5, U$ and $\mathrm{SSH}$ phonon interactions not only both enhance pairing, but their effect is cooperative. This is the central result of our work.

The range of temperatures we can access is limited, but in Fig. 4 we compare $\rho \approx 0.5$ results for $\beta=8$ and $\beta=16$. The temperature dependence of the $4 \times 4$ lattice may not be representative of larger systems because the ground state of the Hubbard model [Eq. (2) with $\lambda=0$ ] on the $4 \times 4$ lattice at $\rho=0.375$ is a triplet for large $U(U \gtrsim 5)$. In the $U=0$ results of Figs. 4(b) and 4(c), at the lower temperature 


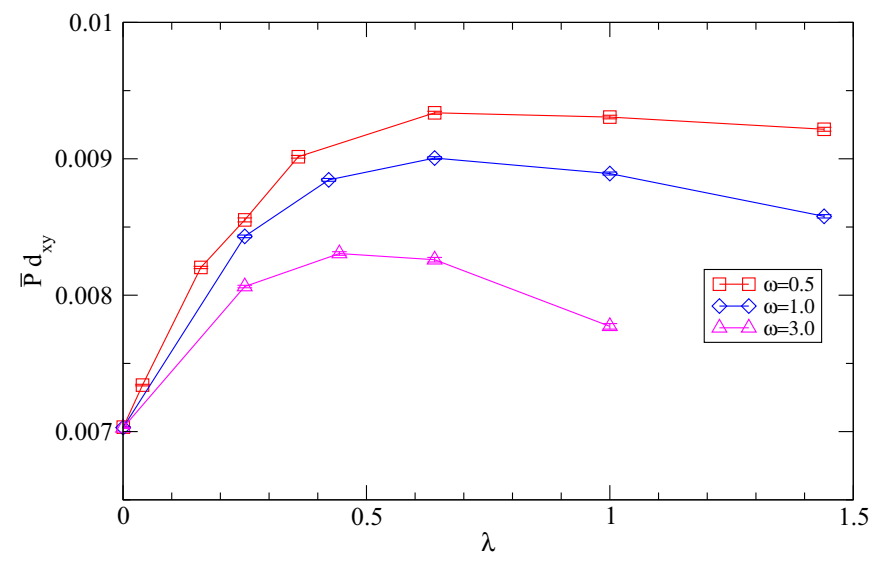

FIG. 5. Plot of $\bar{P}$ versus $e-p$ coupling strength $\lambda$ for $d_{x y}$ pairing in the $6 \times 6$ lattice at $\rho=0.5, \beta=8$, and $U=3$. Squares, diamonds, and triangles correspond to phonon frequencies $\omega$ of $0.5,1.0$, and 3.0 , respectively.

( $\beta=16$ ) the enhancement of $\bar{P}$ by $\lambda$ is somewhat less than at $\beta=8$. This may imply the absence of SC at $U=0$ for $\rho=0.5$ in the ground state of Eq. (2). However, for $U>0$, the amount of enhancement by $U$ at $\lambda \sim \lambda_{\max }$ does increase at $\beta=16$ compared to $\beta=8$. This suggests that the cooperative behavior at $\rho=0.5$ remains at lower temperatures.

Regarding the peak in $\bar{P}$ at $\lambda_{\text {max }}$, a similar broad maximum in $\bar{P}$ is seen as a function of $U$ in zero-temperature $\lambda=0$ calculations at $\rho=0.5[27,28]$. We expect the decrease in $\bar{P}$ at larger $\lambda$ and/or $U$ is caused by the increasing effective mass of pairs. Further, $\lambda_{\max }$ and the amount of enhancement also depend on $\omega$. In Fig. 5 we show the effect of the phonon frequency $\omega$ on $\bar{P}$ for the $6 \times 6$ lattice. As $\omega$ decreases, $\lambda_{\max }$ shifts towards stronger coupling and the amount of enhancement increases. This shows that the phonon dispersion relation will play an important role in understanding a superconducting state mediated by the combination of $e-e$ and $e-p$ interactions.

\section{CONCLUSION}

We have shown that uniquely at $\rho=0.5$ bond-coupled phonons act cooperatively with on-site Coulomb interactions in enhancing superconducting pairing. Both interactions contribute to the superconducting state; the Hubbard $U$ promotes
NN singlet formation, while bond-coupled phonons promote pair hopping. It has been noted that the same pair hopping interaction is present in both the large- $U$ expansion of the bare Hubbard model and the effective interaction $H_{\mathrm{W}}$ [41]. With similar physics contained in both $e-e$ and $e-p$ interaction terms, it then might be expected to find cooperative behavior under the right conditions. Cooperative degrees of freedom are essential to understand phase transitions in real materials including the nonsuperconducting phases of the organic CTS [23]. Producing an unconventional superconducting state from $e-p$ interactions alone in the presence of competing $e-e$ interactions would require a careful tuning of parameters that is unrealistic. As noted above, the best model calculations now suggest that $e-e$ interactions alone are not sufficient for SC. Cooperative $e-e$ and $e-p$ interactions resolve both of these problems.

The observation of cooperative enhancement of pairing only at $\rho \approx 0.5$ supports our proposal of SC emerging from the PEC [23]. The PEC has period-4 charge and bond order lattice $[24,25]$. This is only commensurate on the $4 \times 4$ lattice, where with classical phonons at zero temperature an AFM-PEC transition occurs at a critical $e$ - $p$ coupling strength [24,25]. On the $4 \times 4$ lattice we do not find a strong peak in the bond-bond structure factor suggesting the PEC, but for $\rho=0.5$ the maximum of the bond-bond structure factor is at $\mathbf{Q}=(\pi / 2, \pi)$, which is consistent with the PEC [24,25]. However, with the $\lambda$ and temperatures accessible to DQMC we are not able to reach the PEC transition. Intersite Coulomb interactions $V_{i j}$ (below the critical value $V_{c}$ for Wigner crystal formation) strengthen the PEC and might be necessary to see the PEC state on large lattices [25]. Further calculations over a range of $t^{\prime}$ and on commensurate lattices are beyond the scope of the present work. Studies of the effective interaction $H_{\mathrm{W}}$ across a wider density range will also be useful.

\section{ACKNOWLEDGMENTS}

We thank S. Mazumdar for useful discussions. Some calculations in this work used the Extreme Science and Engineering Discovery Environment [49], which is supported by National Science Foundation Grant No. ACI-1548562. Specifically, we used the Bridges system [50], which is supported by NSF Award No. ACI-1445606, at the Pittsburgh Supercomputing Center under Award No. TG-DMR190068.
[1] G. Ehlers, S. R. White, and R. M. Noack, Hybrid-space density matrix renormalization group study of the doped twodimensional Hubbard model, Phys. Rev. B 95, 125125 (2017).

[2] M. Qin, C.-M. Chung, H. Shi, E. Vitali, C. Hubig, U. Schollwöck, S. R. White, and S. Zhang, Absence of superconductivity in the pure two-dimensional Hubbard model, arXiv:1910.08931.

[3] T. Shirakawa, T. Tohyama, J. Kokalj, S. Sota, and S. Yunoki, Ground-state phase diagram of the triangular lattice Hubbard model by the density-matrix renormalization group method, Phys. Rev. B 96, 205130 (2017).
[4] S. Dayal, R. T. Clay, and S. Mazumdar, Absence of long-range superconducting correlations in the frustrated half-filled-band Hubbard model, Phys. Rev. B 85, 165141 (2012).

[5] D. Reznik, Giant electron-phonon anomaly in doped $\mathrm{La}_{2} \mathrm{CuO}_{4}$ and other cuprates, Adv. Condens. Matter 2010, 523549 (2010).

[6] J. Hofer, K. Conder, T. Sasagawa, G. M. Zhao, M. Willemin, H. Keller, and K. Kishio, Oxygen-Isotope Effect on the InPlane Penetration Depth in Underdoped $\mathrm{La}_{2-x} \mathrm{Sr}_{x} \mathrm{CuO}_{4}$ Single Crystals, Phys. Rev. Lett. 84, 4192 (2000).

[7] M. Bendele, F. von Rohr, Z. Guguchia, E. Pomjakushina, K. Conder, A. Bianconi, A. Simon, A. Bussmann-Holder, and 
H. Keller, Evidence for strong lattice effects as revealed from huge unconventional oxygen isotope effects on the pseudogap temperature in $\mathrm{La}_{2-x} \mathrm{Sr}_{x} \mathrm{CuO}_{4}$, Phys. Rev. B 95, 014514 (2017).

[8] A. Lanzara, P. V. Bogdanov, X. J. Zhou, S. A. Kellar, D. L. Feng, E. D. Lu, T. Yoshida, H. Eisaki, A. Fujimori, K. Kishio, J.-I. Shimoyama, T. Noda, S. Uchida, Z. Hussain, and Z.-X. Shen, Evidence for ubiquitous strong electron-phonon coupling in high-temperature superconductors, Nature (London) 412, 510 (2001).

[9] M. L. Tacon, A. Bosak, S. M. Souliou, G. Dellea, T. Loew, R. Heid, K.-P. Bohnen, G. Ghiringhelli, M. Krisch, and B. Keimer, Inelastic $\mathrm{X}$-ray scattering in $\mathrm{YBa}_{2} \mathrm{Cu}_{3} \mathrm{O}_{6.6}$ reveals giant phonon anomalies and elastic central peak due to charge-density-wave formation, Nat. Phys. 10, 52 (2014).

[10] P. W. Anderson, A suggested $4 \times 4$ structure in underdoped cuprate superconductors: A Wigner supersolid, arXiv:condmat/0406038.

[11] M. Franz, Crystalline electron pairs, Science 305, 1410 (2004).

[12] Z. Tesanovic, Charge Modulation, Spin Response, and Dual Hofstader Butterfly in High- $T_{c}$ Cuprates, Phys. Rev. Lett. 93, 217004 (2004).

[13] H.-D. Chen, O. Vafek, A. Yazdani, and S.-C. Zhang, Pair Density Wave in the Pseudogap State of High Temperature Superconductors, Phys. Rev. Lett. 93, 187002 (2004).

[14] M. Vojta and O. Rösch, Superconducting $d$-wave stripes in cuprates: Valence bond order coexisting with nodal quasiparticles, Phys. Rev. B 77, 094504 (2008).

[15] E. Berg, E. Fradkin, and S. A. Kivelson, Theory of the striped superconductor, Phys. Rev. B 79, 064515 (2009).

[16] P. W. Anderson, The resonating valence bond state in $\mathrm{La}_{2} \mathrm{CuO}_{4}$ and superconductivity, Science 235, 1196 (1987).

[17] A. W. Sandvik, Evidence for Deconfined Quantum Criticality in a Two-Dimensional Heisenberg Model with Four-Spin Interactions, Phys. Rev. Lett. 98, 227202 (2007).

[18] L. Wang and A. W. Sandvik, Critical Level Crossings and Gapless Spin Liquid in the Square-Lattice Spin-1/2 $J_{1}-J_{2}$ Heisenberg Antiferromagnet, Phys. Rev. Lett. 121, 107202 (2018).

[19] H.-C. Jiang, H. Yao, and L. Balents, Spin liquid ground state of the spin- $\frac{1}{2}$ square $J_{1}-J_{2}$ Heisenberg model, Phys. Rev. B 86, 024424 (2012).

[20] W.-Y. Liu, S. Dong, C. Wang, Y. Han, H. An, G.-C. Guo, and L. $\mathrm{He}$, Gapless spin liquid ground state of the spin- $\frac{1}{2} J_{1}-J_{2}$ Heisenberg model on square lattices, Phys. Rev. B 98, 241109(R) (2018).

[21] T. Mizusaki and M. Imada, Gapless quantum spin liquid, stripe, and antiferromagnetic phases in frustrated Hubbard models in two dimensions, Phys. Rev. B 74, 014421 (2006).

[22] N. Gomes, R. T. Clay, and S. Mazumdar, Absence of superconductivity and valence bond order in the Hubbard-Heisenberg model for organic charge-transfer solids, J. Phys.: Condens. Matter 25, 385603 (2013).

[23] R. T. Clay and S. Mazumdar, From charge- and spin-ordering to superconductivity in the organic charge-transfer solids, Phys. Rep. 788, 1 (2019).

[24] H. Li, R. T. Clay, and S. Mazumdar, The paired-electron crystal in the two-dimensional frustrated quarter-filled band, J. Phys.: Condens. Matter 22, 272201 (2010).

[25] S. Dayal, R. T. Clay, H. Li, and S. Mazumdar, Paired electron crystal: Order from frustration in the quarter-filled band, Phys. Rev. B 83, 245106 (2011).
[26] N. Gomes, W. W. De Silva, T. Dutta, R. T. Clay, and S. Mazumdar, Coulomb enhanced superconducting pair correlations in the frustrated quarter-filled band, Phys. Rev. B 93, 165110 (2016).

[27] W. W. De Silva, N. Gomes, S. Mazumdar, and R. T. Clay, Coulomb enhancement of superconducting pair-pair correlations in a $\frac{3}{4}$-filled model for $\kappa$-(BEDT-TTF) ${ }_{2} X$, Phys. Rev. B 93, 205111 (2016).

[28] R. T. Clay, N. Gomes, and S. Mazumdar, Theory of triangular lattice quasi-one-dimensional charge-transfer solids, Phys. Rev. B 100, 115158 (2019).

[29] S. Mazumdar, Valence transition model of the pseudogap, charge order, and superconductivity in electron-doped and holedoped copper oxides, Phys. Rev. B 98, 205153 (2018).

[30] G. R. Stewart, Unconventional superconductivity, Adv. Phys. 66, 75 (2017).

[31] W. P. Su, J. R. Schrieffer, and A. J. Heeger, Solitons in Polyacetylene, Phys. Rev. Lett. 42, 1698 (1979).

[32] M. Hohenadler and H. Fehske, Density waves in strongly correlated quantum chains, Eur. Phys. J. B 91, 204 (2018).

[33] P. Sengupta, A. W. Sandvik, and D. K. Campbell, Peierls transition in the presence of finite-frequency phonons in the onedimensional extended Peierls-Hubbard model at half-filling, Phys. Rev. B 67, 245103 (2003).

[34] R. T. Clay, R. P. Hardikar, and S. Mazumdar, Temperaturedriven transition from the Wigner crystal to the bond-chargedensity wave in the quasi-one-dimensional quarter-filled band, Phys. Rev. B 76, 205118 (2007).

[35] M. Hohenadler, Interplay of Site and Bond Electron-Phonon Coupling in One Dimension, Phys. Rev. Lett. 117, 206404 (2016).

[36] J. Sous, M. Chakraborty, R. V. Krems, and M. Berciu, Light Bipolarons Stabilized by Peierls Electron-Phonon Coupling, Phys. Rev. Lett. 121, 247001 (2018).

[37] S. Li and S. Johnston, Quantum Monte Carlo study of lattice polarons in the two-dimensional multi-orbital Su-SchriefferHeeger model, arXiv:1901.07612.

[38] J. E. Hirsch, Antiferromagnetic singlet pairs, high-frequency phonons, and superconductivity, Phys. Rev. B 35, 8726 (1987).

[39] F. F. Assaad, M. Imada, and D. J. Scalapino, Quantum Transition Between an Antiferromagnetic Mott Insulator and $d_{x^{2}-y^{2}}$ Superconductor in Two Dimensions, Phys. Rev. Lett. 77, 4592 (1996).

[40] F. F. Assaad, M. Imada, and D. J. Scalapino, Charge and spin structures of a $d_{x^{2}-y^{2}}$ superconductor in the proximity of an antiferromagnetic Mott insulator, Phys. Rev. B 56, 15001 (1997).

[41] F. F. Assaad and M. Imada, Doping-induced metal-insulator transition in two-dimensional Hubbard $t-U$ and extended Hubbard $t-U-W$ models, Phys. Rev. B 58, 1845 (1998).

[42] J. E. Gubernatis, N. Kawashima, and P. Werner, Quantum Monte Carlo Methods Algorithms for Lattice Models (Cambridge University Press, Cambridge, 2016).

[43] R. P. Hardikar and R. T. Clay, Phase diagram of the onedimensional Hubbard-Holstein model at half and quarter filling, Phys. Rev. B 75, 245103 (2007).

[44] S. Johnston, E. A. Nowadnick, Y. F. Kung, B. Moritz, R. T. Scalettar, and T. P. Devereaux, Determinant quantum Monte 
Carlo study of the two-dimensional single-band HubbardHolstein model, Phys. Rev. B 87, 235133 (2013).

[45] See Supplemental Material at http://link.aps.org/supplemental/ 10.1103/PhysRevResearch.2.023006 for details.

[46] T. Aimi and M. Imada, Does simple two-dimensional Hubbard model account for high- $T_{c}$ superconductivity in copper oxides?, J. Phys. Soc. Jpn. 76, 113708 (2007).

[47] Z. B. Huang, H. Q. Lin, and J. E. Gubernatis, Quantum Monte Carlo study of spin, charge, and pairing correlations in the $t-t^{\prime}-U$ Hubbard model, Phys. Rev. B 64, 205101 (2001).
[48] M. Laubach, R. Thomale, C. Platt, W. Hanke, and G. Li, Phase diagram of the Hubbard model on the anisotropic triangular lattice, Phys. Rev. B 91, 245125 (2015).

[49] J. Towns, T. Cockerill, M. Dahan, I. Foster, K. Gaither, A. Grimshaw, V. Hazlewood, S. Lathrop, D. Lifka, G. D. Peterson, R. Roskies, J. R. Scott, and N. Wilkins-Diehr, XSEDE: Accelerating scientific discovery, Comput. Sci. Eng. 16, 62 (2014).

[50] N. A. Nystrom, M. J. Levine, R. Z. Roskies, and J. R. Scott, Proceedings of the 2015 XSEDE Conference: Scientific Advancements Enabled by Enhanced Cyberinfrastructure (ACM, New York, 2015), pp. 30:1-30:8. 